- Case Report

\title{
Clival Chordoma in an Adolescent: A Perspective from Primary Care
}

\author{
Hayatul Najaa Miptah', Siti Fatimah Badlishah-Sham¹, Hilwati Hashim², Anis Safura Ramli ${ }^{1,3, *}$ \\ 'Department of Primary Care Medicine, Faculty of Medicine, Universiti Teknologi MARA, Batu Caves, Malaysia \\ ${ }^{2}$ Imaging Unit, Department of Radiology, Faculty of Medicine, Universiti Teknologi MARA, Jalan Hospital, Sungai Buloh, Malaysia \\ ${ }^{3}$ Institute of Pathology, Laboratory and Forensic Medicine, Universiti Teknologi MARA, Sungai Buloh, Malaysia
}

Clival chordoma is a rare malignant tumor of the brain that typically occurs in older adults. It has a high local recurrence rate and is hence associated with poor prognosis. Here, we report a case of an adolescent who presented with a 1-month history of worsening headache and blurring of vision, as well as a 6-month history of left-sided facial and body numbness. Clinical findings were consistent with left upper motor neuron lesion of the seventh cranial nerve with involvement of the fifth cranial nerve. He was also found to have a sixth cranial nerve palsy demonstrated by diplopia upon lateral gaze with no evidence of papilledema. Magnetic resonance imaging of the brain suggested clival chordoma. He was subsequently referred to the neurosurgical team, and he successfully underwent an endoscopic trans-sphenoidal surgery to excise the lesion. He recovered well, continued his follow-ups with the neurosurgical team, and showed good progress. He also attended regular follow-ups with his primary care physician to ensure ongoing psychosocial support and monitoring of his overall health status. This case demonstrates the importance of prompt identification and treatment of clival chordoma in an adolescent. Long-term follow-ups and shared care between primary and secondary care physicians are essential to monitor recurrence of tumor and to provide psychosocial support.

Keywords: Chordoma; Brain Neoplasms; Malignant; Adolescent; Primary Health Care

Received: January 15, 2019, Revised: March 1, 2019, Accepted: March 24, 2019

${ }^{*}$ Corresponding Author: Anis Safura Ramli https://orcid.org/0000-0002-9517-1413

Tel: +603-6126-7463, Fax: +603-6126-7465, E-mail: anis014@uitm.edu.my 


\section{INTRODUCTION}

Clival chordoma is a rare malignant tumor of the skull base that originates from the remnants of the embryonic notochord. ${ }^{1}$ It accounts for $1 \%$ of intracranial tumors and $4 \%$ of primary bone tumors. The incidence rate of chordoma peaked in patients aged $40-50$ years. ${ }^{2)}$ Less than $5 \%$ of all chordoma arising in younger patients were diagnosed in those under 20 years of age. ${ }^{3)}$ Because of its slow growing nature, chordoma usually remains undetected until late stages of the disease. ${ }^{2)}$ The most common presenting symptoms for clival chordoma is diplopia, followed by cranial nerve palsies and headache. ${ }^{2)}$ A previous study reported a low risk for distant metastasis; however, the prognosis is relatively poor as there is a high local recurrent rate with a 5 -year overall survival rate of $73 \%-78 \%$ and 5 -year progression free survival of $50 \%{ }^{2}{ }^{2}$ This case reports an adolescent who was diagnosed with clival chordoma, and discusses the impact of the condition on his life. We also aim to highlight the importance of continuous follow-ups and shared care between primary and secondary care physicians to monitor tumor recurrence and provide long-term psychosocial support to an adolescent.

\section{CASE REPORT}

A 19-year-old male patient presented to the Universiti Teknologi MARA (UiTM) Primary Care Clinic with worsening early morning frontal and parietal headache and blurring of vision over the past month. The headache was described as throbbing in nature, non-radiating, and resolving spontaneously as the day progressed. This was associated with left-sided facial and body numbness, which had gradually worsened over the past 6 months. He did not have nausea, vomiting, history of trauma to the head, or any difficulty in chewing or swallowing. His speech was also unaffected. He only had a small degree of difficulty with tasks involving his left hand such as using keyboards for typing. Otherwise, he could still complete other daily chores as usual. He had no complaints of body weakness and no underlying medical problems prior to the presentation.

The patient is the youngest of six siblings. He currently lives in a suburban area with his parents, both of whom are pensioners. After completing a diploma in religious studies, he worked in the customer service department in a hypermarket for the past year while waiting to enroll for his degree.

Upon examination in the clinic, there was drooping of the left angle of his mouth with flattening of the nasolabial fold. There was no sagging of the eyebrows, and the patient was able to fully close his eyes. Sensation was reduced over the left side of his face. This facial asymmetry had gone unnoticed by the patient himself as well as his family and colleagues at work. These findings were consistent with a left upper motor neuron lesion of the seventh cranial nerve with involvement of the fifth cranial nerve. He was also found to have a sixth cranial nerve palsy demonstrated by diplopia upon lateral gaze with no evidence of papilledema. Other cranial nerves examinations were normal. Neurological examination revealed hyperreflexia over the left upper limb. The power, tone, and sensation were otherwise unaffected.

A magnetic resonance imaging (MRI) scan of the brain revealed a large, solid-cystic pre-pontine mass compressing the pons and fourth ventricle (Figure 1A, B). No evidence of hydrocephalus was observed. There was also a mild cortical involvement of the clivus with minimal bone marrow changes. Subsequently, a computed tomography (CT) scan of the brain revealed erosion of the clivus (Figure 1C). These findings were suggestive of clival chordoma.

The patient was subsequently referred to the neurosurgery team. An endoscopic trans-sphenoidal excision of the lesion was performed. The surgery was otherwise uneventful. The resected tumor was greyish in color. Histopathological examination showed that the tumor tissues were composed of sheets exhibiting small round-to-ovoid nuclei. The cytoplasm was abundant, clear, and vacuolated. They were separated by fibrovascular septae. The tissue was positive for S100, MNF116, and epithelial membrane antigen as demonstrated by immunohistochemical staining. These findings confirmed the diagnosis of clival chordoma. Our patient recovered well in the ward, and was
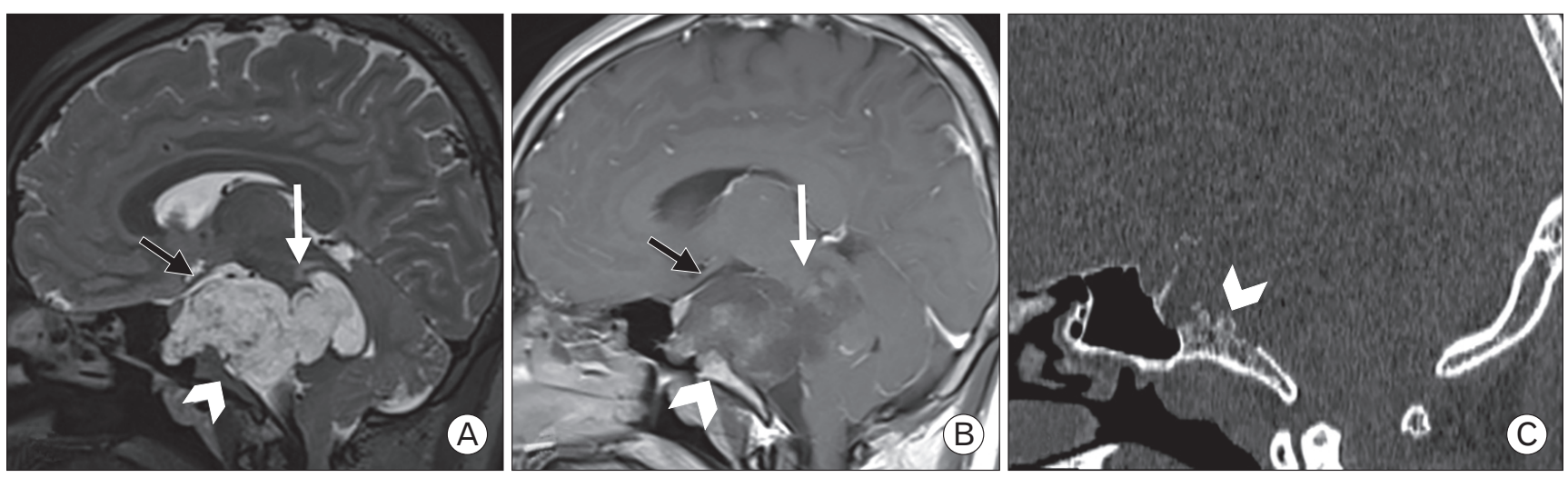

Figure 1. Sagittal magnetic resonance imaging brain demonstrated a lobulated pre-pontine mass (black arrow) which is (A) hyperintense on T2W and (B) non-enhancing postcontrast. The mass is compressing onto the pons (white arrow). It likely arises from the clivus (white arrowhead). (C) Computed tomography brain in bone window demonstrated erosion of the clivus (white arrowhead). Written informed consent for publication of this image was obtained from the patient. 
subsequently discharged on day 7 .

Postoperatively, the patient had a complete resolution of his symptoms. During his 3-month follow-up after the surgery, an MRI scan revealed a reduction in the size of the residual tumor with no evidence of compression or mass effect to the surrounding structures. He was planned for another surveillance MRI to review the progression of the tumor in 6 months.

He is currently being followed-up by the neurosurgical team and the primary care physician. At the neurosurgical follow-up, examinations of his cranial nerves did not reveal any abnormalities, and he was scheduled for brain MRI scans every 6 months to review the progression of the tumor. During his follow-up with his primary care physician, besides the cranial nerve examination, he was also screened for depression and anxiety. These have been negative thus far. Ten months after the initial diagnosis and 6 months postoperatively, he remains asymptomatic.

He receives good support from his family, especially his parents who usually accompany him during the follow-up visits. He has now accepted an offer to study further and is currently doing well in college.

All authors declare that written informed consent was obtained from the patient for publication of this case report and accompanying images.

\section{DISCUSSION}

Brain tumors account for approximately $10 \%$ of all cancers in adolescents aged between 15 to 19 years. ${ }^{4)}$ Chordoma is a rare primary brain tumor; less than $5 \%$ of all cases of chordoma was found arising in patients younger than 20 years of age. ${ }^{3)}$ A chordoma can occur at any location along the axial skeleton, and was found to have an even distribution at the cranial (32\%), spinal (32.8\%), and sacral sites (29.2\%), ${ }^{1)}$ with the most common intracranial site being the clivus.

In the early stages of a brain tumor, patients can remain asymptomatic. ${ }^{2)}$ Our patient had left-sided numbness and facial asymmetry, which was gradually getting worse over 6 months. However, this was unnoticed by his family and friends. He eventually presented with worsening of headache and blurring of vision. Examination findings were consistent with a left upper motor neuron lesion of the fifth, sixth, and seventh cranial nerves. These findings promptly alerted the primary care physician of the possible diagnosis of a brain lesion. His symptoms were consistent with previous studies reporting that in the advanced stages of clival chordoma in young adults, they commonly present with diplopia, headache, and cranial nerve palsies. ${ }^{2)}$ Menezes $^{5)}$ reported a case of a high school student who presented with occipital headache, swallowing difficulty, and dysarthria. She also had hyperreflexia and a broad-based gait. Hanbali et al. ${ }^{6)}$ reported their observation of 24 patients aged 19 years and younger who were diagnosed with skull base tumors including clival chordoma. The most common symptom reported was visual dysfunction. They also reported facial swelling as well as symptoms of sinosanal involvement such as epistaxis as the initial presenting complaint. Furthermore, $20 \%$ of the pa- tients also complained of headaches. ${ }^{6)}$

With regards to imaging modalities chosen for our patient, MRI was suggested by the radiologist as the first-line imaging modality because a brainstem tumor was suspected and also because MRI is highly accessible in our center. Furthermore, MRI is superior to CT when a brainstem tumor is suspected because it can be used to localize the lesion and provide better anatomical detail, less artifacts, and better characterization of the tumor with its high-resolution image. ${ }^{7)}$ A CT scan was subsequently performed to assess bone involvement. However, when access to MRI is limited, CT scan can be used as the initial investigation.

Our patient successfully underwent an endoscopic trans-sphenoidal surgery without any documented complications. This procedure is the current preferred method of removal of lesions from this site. Clival chordoma is thought to be among the most challenging tumors to treat because of its anatomical position. ${ }^{8)}$ In view of that, as well as its high local recurrence rate, Jian et al. ${ }^{8)}$ suggested that radiotherapy postoperatively might increase the success rate to $88.9 \%$. Our patient did not receive any radiotherapy, and this decision was made in view of the almost gross total resection achieved during the procedure. This was decided during the multidisciplinary team meeting between the neurosurgery, oncology, and radiology teams.

Information regarding the recurrence and survival rate of adolescent patients with clival chordoma is essential to predict prognosis of our patient. However, there have been no studies on the recurrence rate of clival chordoma in adolescent patients thus far. A systematic review by Jian et al. ${ }^{8)}$ reported a 5 -year survival rate with the cut-off age of 5 years; patients who were older than 5 years of age were reported to have a higher survival rate. Furthermore, Jian et al. ${ }^{8)}$ did not observe any significant differences in terms of the 5 -year survival rate between patients who underwent surgery alone and patients who received postoperative radiotherapy. Despite advancements in surgery and radiotherapy for patients with clival chordoma, the prognosis has not changed significantly over the years. The 5 -year overall survival rate and 5-year progression free survival have been estimated to be up to $78.4 \%$ and $50.8 \%$, respectively. ${ }^{2)}$ While distant metastases are rare, it has been observed in $7 \%-14 \%$ of cases of chordoma that involved the lungs, liver, bone, and lymph nodes. ${ }^{1)}$

Although our patient is currently symptom-free and doing well psychosocially, he is at risk of developing mental health problems such as depression and anxiety. Therefore, continuous monitoring, appropriate intervention, and support are vital for him to achieve this symptom-free survival. For a primary care physician, caring for survivors of brain tumors or cancer, especially in adolescents, is important to monitor possible cancer recurrence and screen and manage the physical and psychosocial effects impacting the patient. Hudson et al. ${ }^{9}$ has shown that $14.6 \%$ of overall adolescent cancer survivors experienced adverse health effects. The overall prevalence of mental health problems among adolescents and young adults with cancer has been found to range between $16 \%-42 \%$. Patients with cancer in the central nervous system have been reported to have depression and anxiety 
with incidence rates of $19.0 \%$ and $12.1 \%$, respectively. ${ }^{9)}$

In summary, we report a case of a rare primary brain tumor occurring in an adolescent younger than 20 years of age. It is worth noting that patients with clival chordoma will commonly present to their health care providers when symptoms become prominent, which usually is at the advanced stage of the disease. Understanding the nature of the tumor itself is important to identify the red flags as this will lead to early detection, early referral, and hence early management. This knowledge is also helpful in educating the patient and family with regards to treatment and prognosis. Continuous follow-ups and shared care between primary care and secondary care physicians are also essential to provide the patients with ongoing psychosocial support and to monitor for tumor recurrence during their cancer-free survival periods. ${ }^{10)}$

\section{CONFLICT OF INTEREST}

No potential conflict of interest relevant to this article was reported.

\section{ACKNOWLEDGMENTS}

We would like to thank all the clinicians, patient, and family that provided clinical information for this case.

\section{ORCID}

Hayatul Najaa Miptah: https://orcid.org/0000-0001-9756-2621

Siti Fatimah Badlishah-Sham: https://orcid.org/0000-0003-3001-7447

Hilwati Hashim: https://orcid.org/0000-0003-4057-0000
Anis Safura Ramli: https://orcid.org/0000-0002-9517-1413

\section{REFERENCES}

1. Erdem E, Angtuaco EC, Van Hemert R, Park JS, Al-Mefty O. Comprehensive review of intracranial chordoma. Radiographics 2003;23:9951009.

2. Leah P, Dower A, Vescovi C, Mulcahy M, Al Khawaja D. Clinical experience of intracranial chordoma: a systematic review and meta-analysis of the literature. J Clin Neurosci 2018;53:6-12.

3. Hoch BL, Nielsen GP, Liebsch NJ, Rosenberg AE. Base of skull chordomas in children and adolescents: a clinicopathologic study of 73 cases. Am J Surg Pathol 2006;30:811-8.

4. Ward E, DeSantis C, Robbins A, Kohler B, Jemal A. Childhood and adolescent cancer statistics, 2014. CA Cancer J Clin 2014;64:83-103.

5. Menezes AH. Craniovertebral junction neoplasms in the pediatric population. Childs Nerv Syst 2008;24:1173-86.

6. Hanbali F, Tabrizi P, Lang FF, DeMonte F. Tumors of the skull base in children and adolescents. J Neurosurg 2004;100(2 Suppl Pediatrics):169-78.

7. Beltramello A, Lombardo MC, Masotto B, Bricolog A. Imaging of brain stem tumors. Oper Tech Neurosurg 2000;3:87-105.

8. Jian BJ, Bloch OG, Yang I, Han SJ, Aranda D, Parsa AT. A comprehensive analysis of intracranial chordoma and survival: a systematic review. Br J Neurosurg 2011;25:446-53.

9. Hudson MM, Mertens AC, Yasui Y, Hobbie W, Chen H, Gurney JG, et al. Health status of adult long-term survivors of childhood cancer: a report from the Childhood Cancer Survivor Study. JAMA 2003;290: 1583-92.

10. Eilertsen ME, Rannestad T, Indredavik MS, Vik T. Psychosocial health in children and adolescents surviving cancer. Scand J Caring Sci 2011; 25:725-34. 